\title{
Acute Myocardial Infarction due to Coronary Artery Embolism in a Patient with Mechanical Aortic Valve Prosthesis
}

\author{
Marcelo A. Nakazone, ${ }^{1,2}$ Bruno G. Tavares, ${ }^{1}$ Maurício N. Machado, ${ }^{1}$ and Lilia N. Maia ${ }^{1}$ \\ ${ }^{1}$ Department of Cardiology and Cardiovascular Surgery, São José do Rio Preto Medical School, CEP 15090-000, \\ São José do Rio Preto, SP, Brazil \\ ${ }^{2}$ Department of Molecular Biology, São José do Rio Preto Medical School, Av. Brigadeiro Faria Lima 5416, CEP 15090-000, \\ São José do Rio Preto, SP, Brazil
}

Correspondence should be addressed to Marcelo A. Nakazone, naka_med@yahoo.com

Received 29 November 2009; Revised 28 March 2010; Accepted 4 May 2010

Academic Editor: Grigorios Korosoglou

Copyright (C) 2010 Marcelo A. Nakazone et al. This is an open access article distributed under the Creative Commons Attribution License, which permits unrestricted use, distribution, and reproduction in any medium, provided the original work is properly cited.

\begin{abstract}
Previous cases of coronary embolism as a cause of myocardial infarction (MI) in association with prosthetic mechanical valves have been reported, but the fact that the patient was not aware of the importance of maintaining anticoagulation therapy is relevant in this case. A 16-year-old female was referred for primary coronary intervention due to subacute anterolateral ST elevation MI, after she decided to discontinue warfarin therapy three weeks before. Coronary angiography showed distal occlusion of the left anterior descending coronary artery with an image suggesting embolic material. Conventional echocardiography demonstrated akinesia of anteroseptal, inferior, and posterior segments of the left ventricle, with severe systolic dysfunction, beyond the intraventricular thrombus. The presence of mechanic aortic prosthesis and no anticoagulation therapy are highly suggestive of coronary embolism as the cause of MI. This case report confirms that patient education is vital in our struggle to prevent this complication in high-risk patients.
\end{abstract}

\section{Introduction}

Prosthetic mechanical valves carry a significant thromboembolic risk justifying the use of long-term conventional anticoagulation therapy with warfarin [1-3]. The present report describes a case of embolic subacute anterolateral ST elevation myocardial infarction in a younger patient with aortic mechanical valve prosthesis who decided to discontinue warfarin without medical advice.

\section{Case Report}

In October 2009, a 16-year-old female presented to the emergency department with sudden onset of severe chest pain, vomiting, and diaphoresis one day earlier. She had a history of rheumatic aortic stenosis and had received a $23 \mathrm{~mm}$ St. Jude mechanical aortic valve and mitral valve repair four years earlier. Although she had received medical advice regarding constant and imminent thromboembolic risk, she nonetheless decided to abruptly discontinue warfarin and started taking oral contraceptive pills three weeks earlier. She was a smoker but had no other risk factors for coronary artery disease.

At admission, the patient was tachypneic at 25 beats/min with regular rhythm at 107 beats/min. Her blood pressure was 154/92 mmHg. Cardiac auscultation was unchanged with normal prosthetic valve clicks and systolic ejection murmur grade 3/6. No crackles were heard over lungs. Electrocardiography showed left-axis deviation with ST elevation in leads I, aVL, and V5 to V6, compatible with anterolateral wall subacute myocardial infarction (Figure 1). Laboratory tests revealed cardiac troponin I $>50 \mathrm{ng} / \mathrm{mL}$ (upper limit $0.01 \mathrm{ng} / \mathrm{mL}$ ) and RNI of 1.4. Transthoracic echocardiography examination showed normally functioning aortic valve prosthesis with evidence of intraventricular thrombus (Figure 2) and akinesia of the anteroseptal, inferior, and posterior segments of the left ventricle.

Oxygen, acetylsalicylic acid, clopidogrel, and heparin were administered, and the patient was transferred to the catheterization laboratory. Coronary angiography showed a distal occlusion of the left anterior descending coronary 


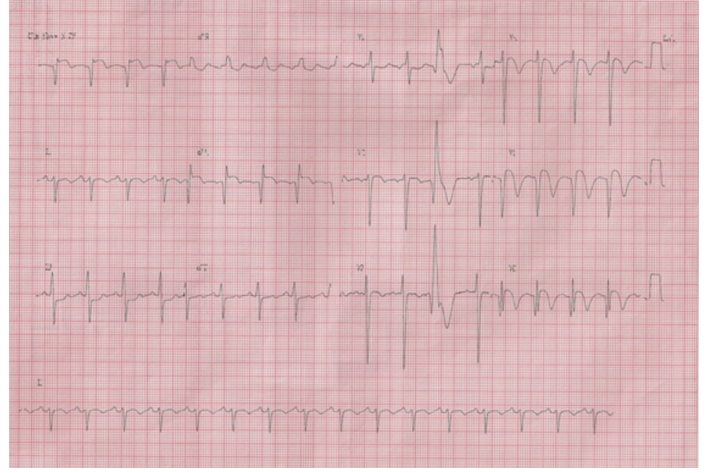

FIGURE 1: Electrocardiography showing left-axis deviation with ST elevation, T-wave inversion and Q-waves in leads I, aVL, and V5 to V6, compatible with subacute anterolateral myocardial infarction and a single ectopic beat.

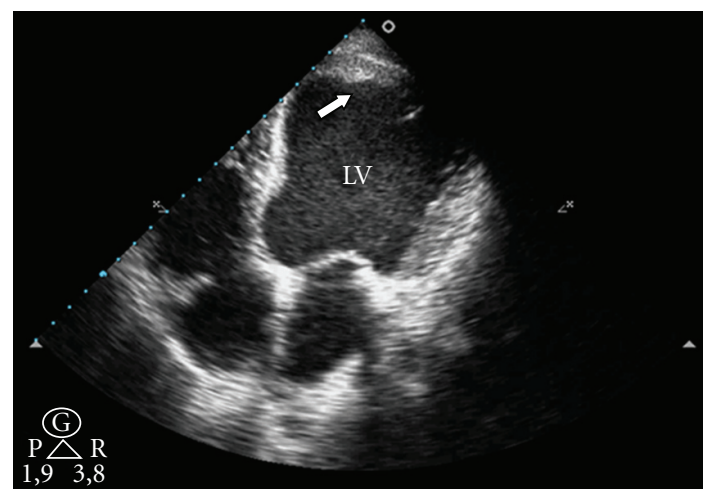

FIgURE 2: Transthoracic echocardiogram image showing thrombus sessile (white arrow) on left ventricular apex (LV).

artery with a concave endoluminal image strongly suggesting embolic material (Figures 3(a) and 3(b)) . The other coronary arteries were normal. Intracoronary catheter aspiration was attempted but was not successful. In addition, percutaneous coronary intervention was attempted but was also unsuccessful due to the distal location of coronary thrombus. We decided not to use thrombolytic therapy because there are fewer published reports and their results are not definitive. On Day 8, a transthoracic echocardiography only showed dyskinesia of the anterior and apical segments of the left ventricle and persistent image suggesting intraventricular thrombus and severe systolic dysfunction. The patient received tirofiban intravenous therapy 48 hours after the procedure. Low molecular weight heparin was administrated following warfarin anticoagulation. The patient was discharged one day later on acetylsalicylic acid, beta-blockers, and angiotensin-converting enzyme inhibitor therapy.

\section{Discussion}

Mechanical heart valves are thrombogenic, and anticoagulation is essential to prevent thromboembolism and acute thrombotic obstruction [2]. Coronary artery embolism is

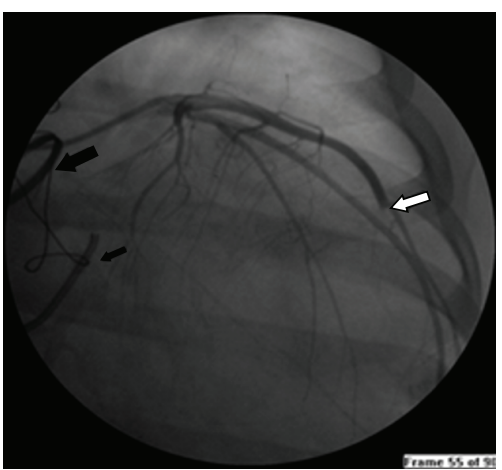

(a)

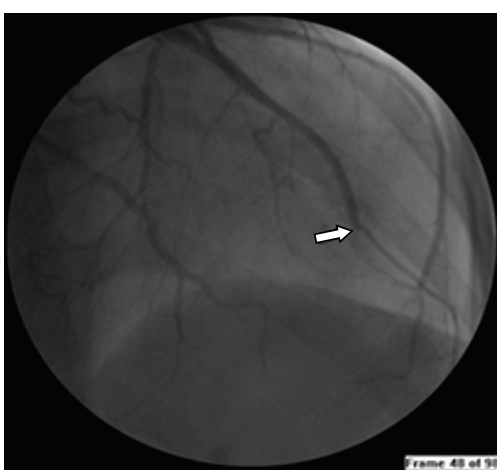

(b)

FIGURE 3: Coronary angiograms showing distal occlusion of the left anterior descending coronary artery with a concave endoluminal image strongly suggesting embolic material in two different projections (white arrows), mechanical aortic valve prosthesis (large black arrow) and mitral valve repair (small black arrow).

not a rare cause of acute transmural myocardial infarction, representing an important entity in terms of etiology and clinical treatment [4], with incidence of 10 to $13 \%$ in autopsy series [5]. The consequences of myocardial infarction secondary to coronary artery embolism depend on both the size of the embolus and the luminal diameter of the vessel $[5,6]$.

Coronary embolism should always be suspected especially in the context of sudden chest pain in patients with valvular prosthesis, chronic atrial fibrillation, dilated cardiomyopathy, infective endocarditis, intracardiac shunts, cardiac myxoma, mural thrombi, and hypercoagulable states $[1,2,7,8]$. It has also been observed that most emboli involve the left coronary system, which could be due to the preferential flow into the artery related to aortic valve morphology [9], as described in our case. However, there is no consensus on the best treatment for coronary embolism.

When a patient presents early with myocardial infarction and ST segment elevation, thrombolysis or percutaneous transluminal coronary angioplasty are two options. Local thrombolysis can be considered but is cumbersome and often ineffective. However, some authors have considered the current medical approach with intravenous thrombolytic therapy $[4,10-12]$. Although there is some evidence that a double regimen with thrombolytic agents is superior to a single regimen, published reports are few and the results are 
not definitive [7]. If the source of the embolus is infective vegetation, thrombolysis is contraindicated. There is a risk of distal embolization causing complete obstruction distally in a smaller branch [5].

Percutaneous transluminal coronary angioplasty and stenting may be used, as reported by Hernández et al. [13] in three interventional cases in which patients with coronary embolism and consequent acute myocardial infarction were treated successfully. In this context, Kiernan et al. [1] reported a 65-year-old male with mechanical aortic valve prosthesis and acute myocardial infarction due to coronary artery embolism. This individual decided to stop taking his warfarin considering previous periods involving bleeding possibly related to interaction between warfarinmetabolizing enzymes and taking a "herbal remedy" for pain causing an inappropriately high INR reading. Differently, in our case, the possible etiology of embolic manifestation was the concomitant presence of multiple thromboembolic risk factors, including smoking, using oral contraceptive pills and discontinued taking of warfarin.

Successful catheter aspiration embolectomy has been described by some authors $[1,7,14,15]$. To prevent distal embolization, Belli et al. [14] and Beran et al. [15] described the use of commercial systems to aspirate thrombus from a native coronary artery and to improve the safety of epicardial flow. In our patient, catheter aspiration embolectomy for the intracoronary embolism was not effective. The complete distal obstruction of the left anterior descending coronary artery turned this procedure unfeasible. Still, the location of the coronary thrombus was a challenging place to reach and treat using percutaneous coronary intervention. In this case, anticoagulation with heparin and intravenous glycoprotein inhibitors was performed. This may not result in thrombus resolution but may prevent further increase in thrombus size [5]. It is also important to emphasize the need for aggressive anticoagulation, particularly in patients with mechanical prostheses, according to ACC/AHA guidelines [16].

The present report described a case of acute myocardial infarction due to coronary artery embolism in a smoker patient with mechanical aortic valve prosthesis who decided to abruptly discontinue her warfarin and started taking oral contraceptive pills three weeks earlier. It illustrates, once again, the risks of embolic events in high-risk patients, alerting for the hypothesis of embolic myocardial infarction in similar situations. In this context, the patient education on the optimal anticoagulation with warfarin and the medical advice about imminent thromboembolic risks are of extreme importance.

\section{References}

[1] T. J. Kiernan, A. M. O. Flynn, and P. Kearney, "Coronary embolism causing myocardial infarction in a patient with mechanical aortic valve prosthesis," International Journal of Cardiology, vol. 112, no. 2, pp. E14-E16, 2006.

[2] J.-P. Lavoie, L. Leduc, and L.-A. Mercier, "Embolic myocardial infarction in a pregnant woman with a mechanical heart valve on low molecular weight heparin," Canadian Journal of Cardiology, vol. 20, no. 9, pp. 917-919, 2004.
[3] P. Varadarajan, D. Isaeff, and R. G. Pai, "Prosthetic valve thrombosis presenting as an acute embolic myocardial infarction in a pregnant patient: issues on anticoagulation regimens and thrombolytic therapy," Echocardiography, vol. 23, no. 9, pp. 774-779, 2006.

[4] M. Dogan, S. Acikel, M. M. N. Aksoy, et al., "Coronary saddle embolism causing myocardial infarction in a patient with mechanical mitral valve prosthesis: treatment with thrombolytic therapy," International Journal of Cardiology, vol. 135, no. 2, pp. e47-e48, 2009.

[5] M. S. Aslam, V. Sanghi, S. Hersh, and J. B. Lakier, "Coronary artery saddle embolus and myocardial infarction in a patient with prosthetic mitral valve," Catheterization and Cardiovascular Interventions, vol. 57, no. 3, pp. 367-370, 2002.

[6] B. F. Waller, D. S. Dixon, R. W. Kim, and W. C. Roberts, "Embolus to the left main coronary artery," American Journal of Cardiology, vol. 50, no. 3, pp. 658-660, 1982.

[7] W.-C. Hung, C.-J. Wu, W.-J. Chen, C.-H. Yang, and J.-P. Chang, "Transradial intracoronary catheter-aspiration embolectomy: for acute coronary embolism after mitral valve replacement," Texas Heart Institute Journal, vol. 30, no. 4, pp. 316-318, 2003.

[8] P. A. Kraus and J. Lipman, "Coronary embolism causing myocardial infarction," Intensive Care Medicine, vol. 16, no. 3, pp. 215-216, 1990.

[9] K. R. Prizel, G. M. Hutchins, and B. H. Bulkley, "Coronary artery embolism and myocardial infarction. A clinicopathologic study of 55 patients," Annals of Internal Medicine, vol. 88, no. 2, pp. 155-161, 1978.

[10] C. Steinwender, R. Hofmann, B. Hartenthaler, and F. Leisch, "Resolution of a coronary embolus by intravenous application of bivalirudin," International Journal of Cardiology, vol. 132, no. 3, pp. e115-e116, 2009.

[11] Y. Atmaca, C. Ozdol, and C. Erol, "Coronary embolism in a patient with mitral valve prosthesis: successful management with tirofiban and half-dose tissue-type plasminogen activator," Chinese Medical Journal, vol. 120, no. 24, pp. 2321-2322, 2007.

[12] E. G. Quinn and D. J. G. Fergusson, "Coronary embolism following aortic and mitral valve replacement: successful management with abciximab and urokinase," Catheterization and Cardiovascular Diagnosis, vol. 43, no. 4, pp. 457-459, 1998.

[13] F. Hernández, M. Pombo, R. Dalmau, et al., "Acute coronary embolism: angiographic diagnosis and treatment with primary angioplasty," Catheterization and Cardiovascular Interventions, vol. 55, no. 4, pp. 491-494, 2002.

[14] G. Belli, A. Pezzano, A. M. De Biase, et al., "Adjunctive thrombus aspiration and mechanical protection from distal embolization in primary percutaneous intervention for acute myocardial infarction," Catheterization and Cardiovascular Interventions, vol. 50, no. 3, pp. 362-370, 2000.

[15] G. Beran, I. Lang, W. Schreiber, et al., "Intracoronary thrombectomy with the X-sizer catheter system improves epicardial flow and accelerates ST-segment resolution in patients with acute coronary syndrome: a prospective, randomized, controlled study," Circulation, vol. 105, no. 20, pp. 2355-2360, 2002.

[16] R. O. Bonow, B. A. Carabello, K. Chetterjee, et al., "2008 focused update incorporated into the ACC/AHA 2006 guidelines for the management of patients with valvular heart disease: a report of the American College of Cardiology/American Heart Association Task Force on Practice 
Guidelines (Writing Committee to Revise the 1998 Guidelines for the Management of Patients With Valvular Heart Disease): endorsed by the Society of Cardiovascular Anesthesiologists, Society for Cardiovascular Angiography and Interventions, and Society of Thoracic Surgeons," Journal of the American College of Cardiology, vol. 52, no. 13, pp. e1-e142, 2008. 


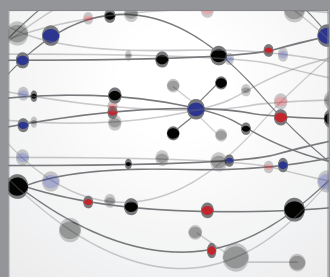

The Scientific World Journal
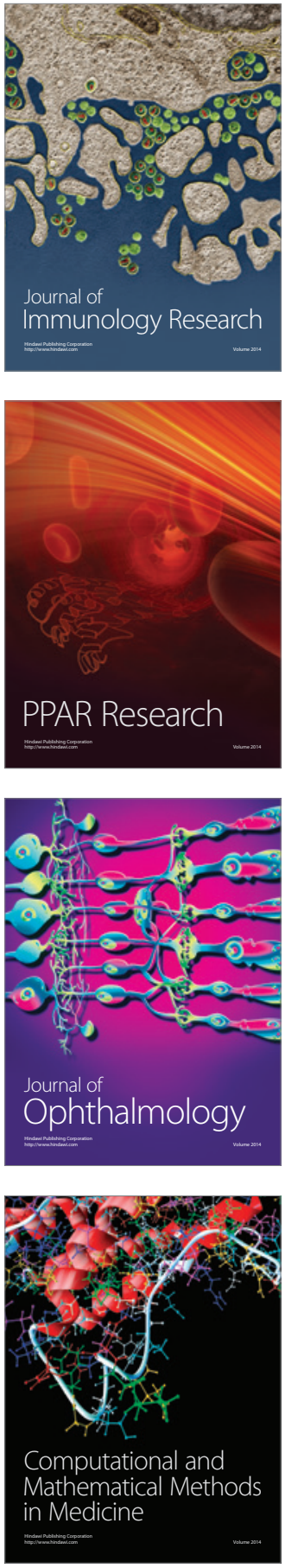

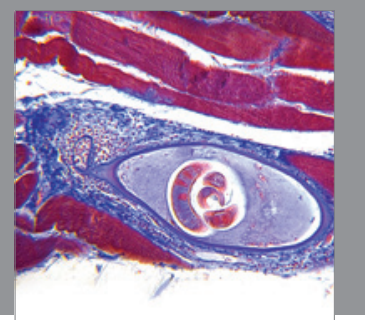

Gastroenterology

Research and Practice
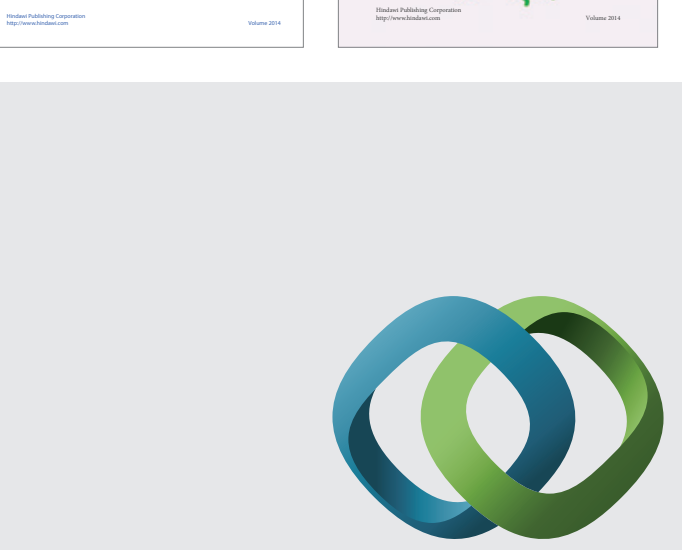

\section{Hindawi}

Submit your manuscripts at

http://www.hindawi.com
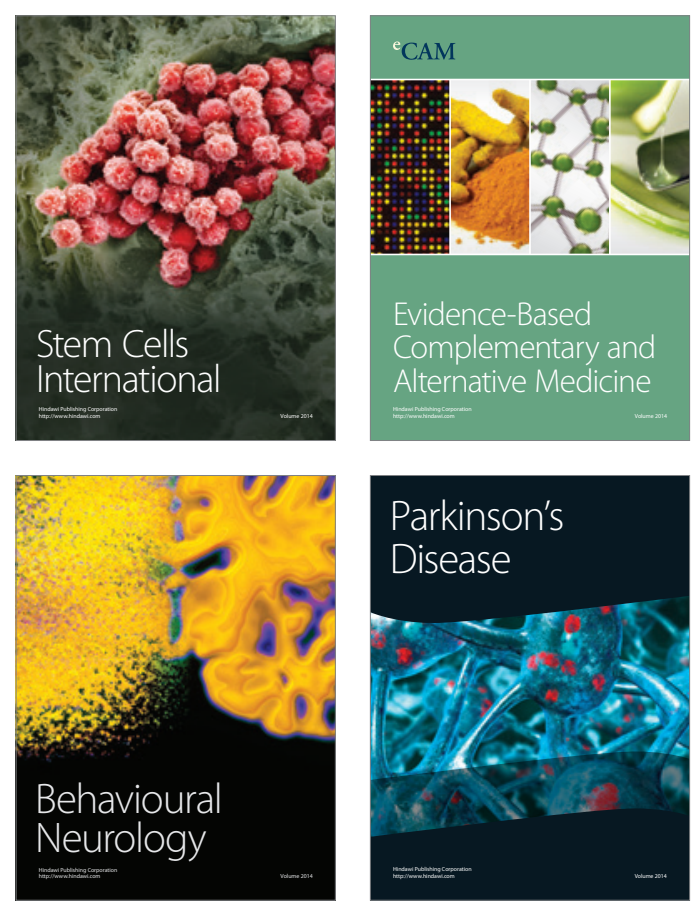

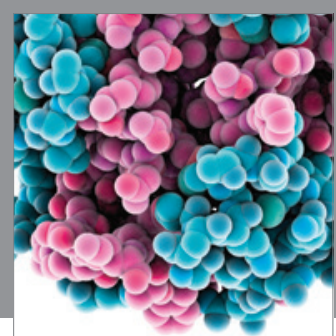

Journal of
Diabetes Research

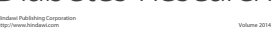

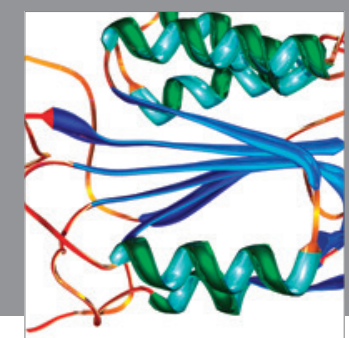

Disease Markers
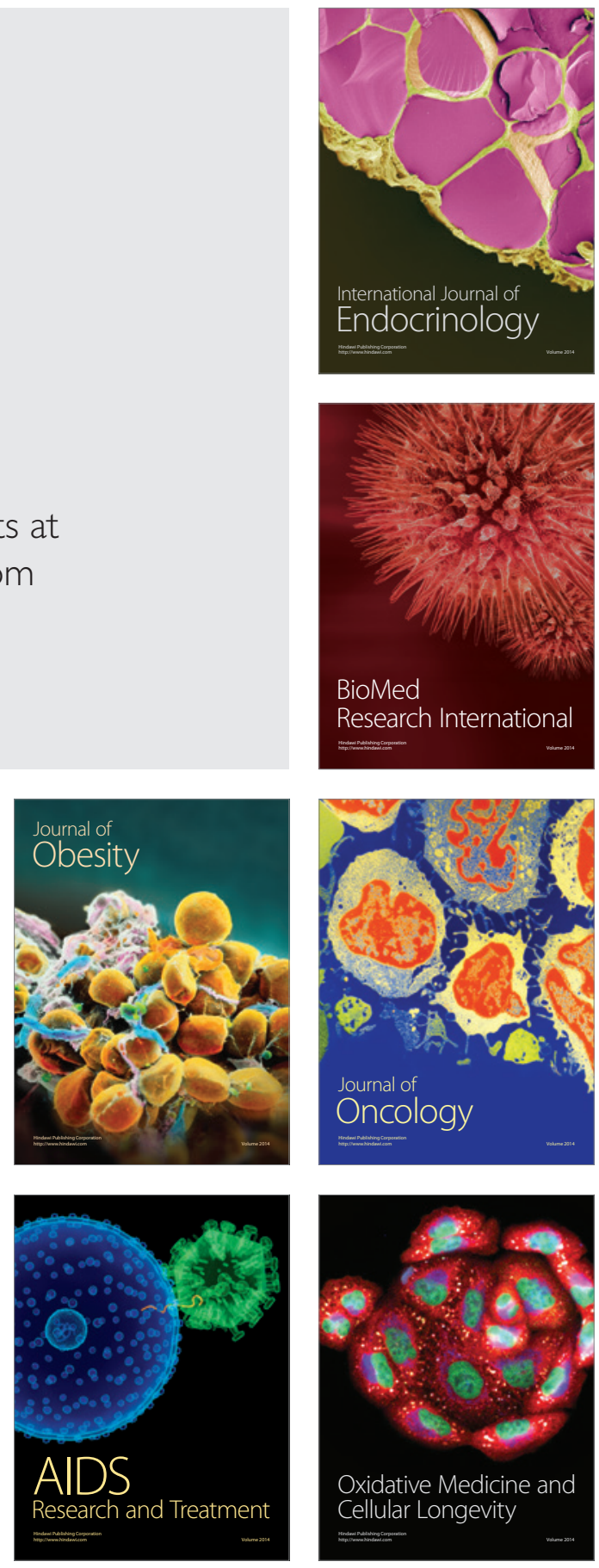